\title{
Mars as a "natural laboratory" for studying surface activity on a range of planetary bodies
}

Corresponding Author: Serina Diniega (Jet Propulsion Laboratory, California Institute of Technology (JPL); serina.diniega@jpl.nasa.gov; 626-720-7293)

Co-authors: Ali Bramson (Purdue U), Bonnie Buratti (JPL), Peter Buhler (JPL), Devon Burr (N Arizona U), Matt Chojnacki (PSI), Susan Conway (U Nantes), Ingrid Daubar (Brown U), Cynthia L. Dinwiddie (SwRI), Anna Grau Galofre (ASU), Brian Jackson (Boise State U), Alfred McEwen (U Arizona), Mathieu Lapôtre (Stanford U), Joseph Levy (Colgate College), Lauren McKeown (Nat History Museum), Sylvain Piqueux (JPL), Anya Portyankina (CU Boulder), Scot Rafkin (SwRI), Simone Silvestro (INAF), Isaac Smith (York U \& PSI), Christy Swann (Naval Res Lab), Jacob Widmer

Acknowledgements: A portion of the work described was carried out at the Jet Propulsion Laboratory, California Institute of Technology, under a contract with the National Aeronautics and Space Administration (80NM0018D0004).

Signatories: Amy C. McAdam (NASA GSFC), Pat Becerra (U Bern), Shane Byrne (U Arizona), Mackenzie Day (UCLA), Taylor Dorn (UCLA), Colin Dundas (U.S. Geological Survey), Ryan C. Ewing (Texas A\&M U), Timothy A. Goudge (U Texas, Austin), Amber Gullikson (U.S. Geological Survey), Shannon Hibbard (U West Ontario), Cecilia Leung (JPL), Tim I. Michaels (SETI Inst), Scott L. Murchie (JHU/APL), Rodriguez Sebastien (U Paris, IPGP), Hanna Sizemore (PSI), Robert Sullivan (Cornell U), Timothy Titus (U.S. Geological Survey)

\section{Executive Summary}

This white paper demonstrates: Mars is a uniquely enabling study target for investigations of surface processes active on other planetary bodies - both for extending terrestrial based process models and, in some areas, for serving as a superior comparative planetology basis over Earth. Recommendations are made for specific future measurement types and for investments in technology, complementary research, and workforce development to optimize the value of Mars observations within planetary science geologic and climatologic interpretations from surface features and activities.

(C) 2020. All rights reserved.

Submitted to the Planetary Science \& Astrobiology Decadal Survey, 2023-2032 


\section{Mars is a prime target for observing and investigating active surface processes}

Mars is a great target for active surface process investigations as:

- Repeat high-resolution observations have yielded many examples of present-day surface activity [White Paper/WP: Dundas et al.]. These current changes have or could be studied in enough detail to identify the driving process(es) and/or influential environmental conditions.

- Mars' surface activity in modern times and through the last $~ 2$ Gyrs can be used as a comparative planetology basis for (1) processes active on Earth, but under very different conditions (e.g., aeolian processes within a low density atmosphere or with much higher obliquity), and (2) processes not active on Earth but that are active on other planetary bodies (e.g., sublimation-dominant frost dynamics; or records of active processes without complications from recent extensive fluvial or biological activity).

- Mars' atmospheric, surface, and planetary conditions are different enough from Earth's to test and stretch terrestrial study-based models, but similar enough that the terrestrial models are a reasonable starting point. As will be described, Mars' conditions often have values between those on Earth and other planetary bodies (such as KBOs or the Moon).

- Decades of previous investment have yielded ample geologic and atmospheric contextual information and models that will greatly enhance incorporation of the broader geologic and atmospheric/climatological context within process-focused investigations.

- There may be relatively many future opportunities for gathering new data needed to constrain/calibrate process models. Commercial and international interest in sending spacecraft (and humans) to Mars along with Mars' relatively close proximity to Earth suggest that access opportunities should exist over the next decade, especially for small spacecraft that do not need a dedicated launch vehicle [WP: Barba et al.] — which may be sufficient for targeted environmental monitoring studies [e.g., WP: Diniega et al.].

\section{Areas in which studies of Mars' environmental conditions \& processes enable testing of} models of analogous processes on other planetary bodies Due to factors listed in Box 1, studies of the surface processes involved in martian landform and landscape morphology and evolution can be viewed through a "natural laboratory"-investigation lens and used to advance our understanding of other planetary bodies. In particular, we discuss (a) aeolian surface processes and meteorological dynamics; (b) sublimation-driven dynamics, (c) impact cratering rates and processes, and (d) variable density atmospheres. (At Mars, one can also study both the above individual phenomena and interactions between them.) Beyond these studies, there are many study areas where Mars serves well as a non-Earth comparison point or extraterrestrial "laboratory" for testing and refining models: e.g., habitability/life evolution [WP: Cabrol et al.; Jakosky et al.], atmospheric dynamics [WP: Tamppari et al.], and climate records [WP: Becerra et al.; Smith et al.].

\section{a) Aeolian surface processes \& meteorological dynamics}

Aeolian (wind-driven) sand and dust are known to significantly influence landscape evolution and climate across the solar system [WP: Newman et al.; Titus et al.]. In addition to the phenomena studied on Earth and Mars:

Box 1. Mars' characteristics that enable its studies to bridge the gap between terrestrial-based models and perspectives to studies of all types of planetary bodies include:

- Low gravity (but not very low)

- Low (and varying) atmospheric density

- Climate cycles

- A primarily $\mathrm{CO}_{2}$ and sublimation-driven seasonal volatile cycle (i.e., little liquid)

- Sublimation of frost

- Low/zero amount of recent fluvial activity

- Likely low/zero amount of recent biological activity

- Measureable impact rates and crater evolution in wellpreserved/characterized terrains 
- A few dune fields ${ }^{1,2}$, many wind streaks, and a few potential yardangs ${ }^{2}$ have been identified on Venus, under an atmosphere 9× thicker than Earth's.

- On Saturn's moon Titan, sand produced from photochemical aerosols and water ice has formed vast dunes and sand seas ${ }^{3,4}$; dust storms may also occur during equinox ${ }^{5}$.

- Even on comets ${ }^{6}$ and icy worlds, such as Pluto ${ }^{7}$, aeolian processes within a transient, very thin atmosphere appear to have formed bedforms.

Interpretations of these landforms and processes has generally relied upon models of sediment fluxes and transport dynamics (i.e., saltation and reputation rates and profiles) that are primarily based on terrestrial field and laboratory experiments, with scaling applied based on specific planetary conditions. This has led to new tests of bedform evolution models and proposal of new dynamics regimes ${ }^{8-14}$ after the predicted scaling for another planet is found to be inconsistent with the Earth conditions-based model.

Further study of martian aeolian and other meteorological systems, and how conditions drive surface activity, will enable more detailed testing and refinement of surface-atmosphere interaction process models. In particular, in situ "field" measurements of martian atmospheric boundary layer dynamics driving sand/dust and volatile transport would provide novel calibration data for models and wind-tunnel experiments [WP: Diniega et al.; Burr et al.] within an environment with a substantially lower impact threshold than fluid threshold ${ }^{9}$. Such "ground truth" is needed to advance a cross-planet model for, e.g., sand and dust lofting and transport. Another model presently untested under extraterrestrial conditions is the one used to estimate turbulent eddy fluxes (which result in the exchange of energy, momentum, and quantities like dust, water, and other chemical species between the surface and atmosphere). On Earth, turbulent fluxes can be directly calculated from correlated, high frequency measurements of the 3-D wind components and the quantity of interest; such fluxes are also related to large-scale (and more easily measured) quantities ${ }^{15-17}$ such as the vertical gradient of temperature and of the horizontal wind (i.e., the wind shear), with ample testing and calibration of the physical model through terrestrial field and laboratory studies. These relationships are assumed in planetary studies, but have never been shown to extend to those environments despite generally being far outside of terrestrial conditions (e.g., Mars has an extremely stable nocturnal inversion and unstable afternoon convective layer). Such measurements can also contribute to characterization of Planetary Boundary Layer dynamics (especially through combination with orbital data)—potentially generating science and methodology overlap with high-priority terrestrial studies [2017 Earth Science Decadal Survey].

Mars is already used as the comparative planetology basis for some studies. For example, the threshold curve under low-density gas conditions derived for Mars in the NASA Ames Wind Tunnel (MARSWIT) $)^{18,19}$ has also been used to model aeolian-type transport resulting from jetting on comets ${ }^{20}$. Images from the New Horizons mission showed that Pluto has decameter-wavelength "bedform" morphologies that have been proposed to be aeolian dunes". Based on these bedform measurements, a minimum wind shear required for saltation on Pluto was estimated based on work performed in the MARSWIT that separated Reynolds number and interparticle cohesion effects ${ }^{21}$.

\section{b) Sublimation-driven dynamics}

The $\mathrm{CO}_{2}$ atmosphere of Mars is in vapor pressure equilibrium with surface ice; seasonally $\mathrm{CO}_{2}$ sublimates and condenses, changing the atmospheric density by $>25 \%$. Mars has lower gravity and a lower pressure and temperature environment than Earth, causing sublimation processes to differ from terrestrial analogs, including laboratory analogs. Mars, therefore, has a more similar environment to Triton, Pluto, and other Kuiper Belt Objects (KBOs) than Earth and laboratory analogs. For example, no large-scale sublimation dynamics naturally occur on Earth, and within laboratory studies we cannot replicate low gravity over timescales relevant for sublimation. 
Sublimation is thought to be the main driver forming a range of depressions or pits on Mars. Different features are proposed to form due to sublimation from volatile reservoirs of different sizes/forms/ages, and vastly different sublimation rates. Studies of these features on Mars enables discrimination between different proposed models and identification of key geomorphological or environmental signatures for separating features that likely formed through slightly different processes, within different environments, and/or over very different timescales. For example:

- South polar swiss cheese and north polar cap morphologies are proposed to be formed through short-term cyclic (i.e., seasonal) sublimation of a large surface volatile reservoir. Patterned ground is proposed to be formed through cyclic sublimation of a mixed subsurface volatile reservoir. Mid-latitude icy scarps and scalloped depressions are proposed to be formed through long-term sublimation of a large subsurface volatile reservoir. (For many references, see recent community summaries ${ }^{22-24}$ ).

- Pitted terrain in and around impact craters is proposed to be formed due to sudden sublimation of impact-heated, buried volatiles ${ }^{25,26}$.

These types of depressions and pits offer analogs:

- Hollows on Mercury ${ }^{27,28}$ which are thought to be due to volatile-loss or sublimation of sulfurrelated compounds (likely sulfides) and analogies have been drawn to swiss cheese terrain.

- Pitted surfaces have been discovered on asteroids; notably, pitted crater floors on Vesta ${ }^{29}$ and Ceres $^{30}$ are remarkably similar to pitted terrain on Mars. Pitted terrains on Pluto are thought to represent erosion from sublimation-driven winds of the surface of nitrogen ice and possibly methane ice ${ }^{31}$. Similar terrains are thought to exist on other Kuiper Belt Objects ${ }^{32}$, including Arrokoth $^{33}$. Pitted surfaces are found on comets ${ }^{34}$ and recent exploration of 67P ChuryumovGerasimenko maintains that their growth is dominated by sublimation ${ }^{35,36}$.

On Mars, sublimation is also thought to have an important role to play in downslope mass transport, namely on sand dunes and within the context of gullies [WP: Dundas et al.]. Such systems could form useful analogies for:

- Gully-like landforms identified on Mercury ${ }^{37}$ and Vesta ${ }^{38,39}$, potentially related to sublimation of sulfur-compounds and water, respectively.

- Downslope features that have been observed on Helene, one of Saturn's Trojan moons, whose formation has been ascribed to sublimation ${ }^{40}$.

Furthermore, it is likely that future close inspection of other solar system bodies will reveal more examples of sublimation-driven mass-wasting processes.

Escaping pressurized gas from sublimation at the base of the $\mathrm{CO}_{2}$ seasonal ice cap on Mars erodes araneiforms ${ }^{41,42}$, radially-organized and /or dendritic channels ${ }^{43}$ initially dubbed "spiders", and deposits patterned material across the surface ${ }^{44}$. These jets of gas, whose origin is via basal sublimation and the solid state greenhouse effect ${ }^{45}$, and the patterns/rates of material they spew out could be useful analogues for:

- Solar-driven jets of materials found on other bodies, notably Triton (and the solar-driven theory was proposed to explain plumes on Triton ${ }^{46}$ before it was applied to Mars' exogenic geysers).

- Global-scale contrasting albedo regions on Iapetus and Ganymede ${ }^{47-49}$.

- $\mathrm{CO}_{2}$ ice signatures found on the trailing hemispheres' of the Uranian satellites ${ }^{50}$, notably visible as a bright deposit inside Wunda Crater on Umbriel ${ }^{51}$. Severe sublimation of $\mathrm{CO}_{2}$-ices is thought to explain the pinnacle terrain on Callisto ${ }^{52,53}$.

While not directly related to sublimation, the creep of martian glaciers also likely would be a useful analog for studies of outer Solar System bodies. The balance between sublimation/ablation, deposition, and flow rates would be significantly different in martian vs. terrestrial glaciers. In particular, due to an overall lower surface temperature, water-ice glaciers on Mars exhibit different dynamics from most terrestrial glaciers, i.e., without basal melting or basal sliding ${ }^{54,55}$ and 
potential existence of past $\mathrm{CO}_{2}$ glaciers ${ }^{56}$. Whether martian polar layered deposits exhibit any significant flow behavior is still an open question [WP: Smith et al.], and it has been proposed that stratified layers of different materials with different viscous properties may prevent flow at large scales $^{57}$. Such behaviors are unlike those observed within any large ice reservoirs on Earth, but may be analogous to behaviors occurring in the outer Solar System or even outside of the Solar Sytem $^{58}$. Even if not providing a direct analog, study of Mars ices and related behaviors/landforms may help ground truth flow models and connect with experiments involving exotic ices.

\section{c) Impact cratering rates}

In the last decade, current impact cratering has been observed on Mars ${ }^{59,60}$ as well as on the Moon $^{61}$. Detailed studies of new craters on these bodies yield information about the impact cratering process at planetary scales in terrestrial-like target materials, without the interference of a thick terrestrial atmosphere or the confounding effects of weathering, erosion, and secondary cratering. Like the Moon, Mars' atmosphere is thin enough to not completely block small impacts (as occurs on Earth). Unlike the Moon, Mars' thin atmosphere is enough to demonstrate some atmospheric effects on cratering: fragmentation, deceleration, and ablation. Thus, Mars' craters and impact rates can be compared to the Moon's to differentiate effects of the atmosphere from observational biases or effects of differing gravity or secondary impact cratering.

Additionally, Mars' location near the asteroid belt lets it act as a "witness plate”, capturing a sample of the main belt small-body population that cannot currently be observed directly. This allows for interplanetary comparisons of the current impact flux, opening the way for deeper studies of differences between the overall fluxes, size distributions, sources (asteroidal or cometary), and dynamics of the impacting population at the Earth/Moon system compared to Mars, and eventually providing better constraints for extrapolation to impact rates on other planetary bodies. These measured impact rates are also leading to refinements of standard chronology models $^{62}$ applied throughout the inner Solar System. Furthermore, measurements of short-term variability in the impact rate will illuminate orbital dynamics of Mars and its impacting population, and serve as a comparison for the Earth/Moon system where variability is expected to be due to showers from passing comets ${ }^{63}$, as opposed to seasonal variations in orbital distance.

\section{d) Variable-density atmospheres}

Seasonal variations in martian atmospheric pressure are directly driven by the sublimation of seasonal $\mathrm{CO}_{2}$ ice layer in the winter/spring hemisphere and its deposition in the fall/winter hemisphere. The dynamics of this process modulates the global climate circulation and drives local sublimation winds, such as katabatic winds that are thought to play an important role in the formation of polar troughs ${ }^{64}$. Similar surface-atmosphere processes act on other planetary bodies where atmosphere is in vapor pressure equilibrium with surface ice, such as on $\mathrm{KBOs}^{65,66}$.

Due to cycles in various orbital parameters (such as obliquity), the Mars atmospheric density may cycle through a range of 1-12 mbar over thousands to millions of years timescales ${ }^{67,68}$. Derivation of present-day martian surface activity models that quantitatively connect landform morphologies to driving environmental conditions will enable improved interpretation of relict features and reduce uncertainty when extrapolating activity models through past Mars climates. Such developments will also provide a testable basis for generation of similar models on other bodies that also experience large, cyclic changes in atmospheric density, such as on Pluto ${ }^{64}$ and Triton $^{69}$. As Earth's atmosphere has not gone through comparable large swings in atmospheric density during the portion of Earth's history when most of the Earth's observable rock record was formed, Mars provides important "ground truth" for this type of extrapolative analysis and integration of predicted geologic records through different atmospheric pressures. 
In parallel, but out of phase with variable density atmospheres, the surface deposition of meteoric ice will result in layered and likely stratified volatile deposits with impurities. On Mars, impurities likely include dust, lithic fragments from volcanic eruptions or ejecta, fine salt grains, trapped gasses, and isotopologues ${ }^{22,70}$ [WP: Becerra et al.]. Mars is not the only planetary body to experience partial atmospheric collapse ${ }^{71}$. Pluto ${ }^{72-74}$ and Titan $^{75}$ likewise have strong seasonal (hundreds of years) atmospheric cycles and orbital variations that could cause similar ice layering as is found on Mars [WP: Smith et al.], and atmospheric collapse has been proposed for tidallylocked planets around TRAPPIST- ${ }^{58}$. Earth, with anthropomorphic influences, abundant biology, and liquid-phases, does not provide a good analog for such layered ice deposits or climate models.

\section{Highest-priority planetary surface science questions and spacecraft measurements}

The general aim of geomorphic studies is to quantitatively connect observed landforms to their formative environmental drivers via models of the active process(es). For the landforms and surface activity we have described, advancements generally require more complete information about the specific environmental drivers for formation and subsequent modification/evolution. For example, more complete knowledge about how and where frozen $\mathrm{CO}_{2}$ accumulates, and how frost/ice layers evolves through the cold seasons, would provide important constraints on models of continued monitoring of global atmospheric conditions and dynamics (including winds, clouds, and dust content), coupled with information about surface properties are needed to constrain models of $\mathrm{CO}_{2}$ basal sublimation (or the Kieffer effect ${ }^{45}$ ) and identify the controlling factors that determine the scale of the eruptive vents and basal erosion that lead to araneiform activity. Such information, compared with observations of the growth of the seasonal ice cap, would also allow for tests of predictions of where $\mathrm{CO}_{2}$ ice is of sufficient thickness and strength for the formation and growth of araneiforms - in the present climate or during a recent past climate. Important information can be gathered through some complementary studies (outlined in Box 2).

To acquire observational data related to mapping and timing of activity, continued highresolution orbital imagery is key. The advent of HiRISE-type imaging demonstrated that the martian surface is active in the present-climate, yielding a paradigm shift from the view that all the interesting martian geologic activity occurred in the ancient past [WP: Dundas et al.]. Continued repeat imaging of the surface, ideally with similar sub-meter resolution and lighting as HiRISE images, would enable identification of yet more surface changes, including those with slower activity rates, and timing/environmental constraints. Interannual variations in surface activity can also be tracked, yielding another way to constrain driving environmental drivers. Increased spatial coverage will enhance mapping studies (to date, HiRISE has imaged only 2\% of the globe). If the focus is to constrain activity to specific times of day, an orbit would be needed that will drift through local solar time (such as that proposed in NEX-SAG ${ }^{76}$ ). Alternatively, in situ observations can provide detailed timing constraints on local activity [WP: Diniega et al.].

To connect the landforms to environmental controls, we also need measurements of the environment where these landforms and activity are found. Coupling surface and subsurface

Box 2. With observational data:

1) Mapping where the landforms exist and/or are active, and where they are not.

2) Geomorphological measurement of the landform and its activity.

3) Characterization of the timing of activity (e.g., by season, by time of day, and identification of interannual variation).

4) Characterization of the surface (and potentially subsurface) and atmospheric environment where and when the activity occurs.

With laboratory, terrestrial field analog, and physics modeling studies:

5) Identification of possible environmental drivers and investigation of scaling relationships/temporal evolution rates. 
compositional, thermophysical, and structural measurements with meteorological conditions over sites where a specific landform and/or activity is observed allows for a holistic look at the full system. From orbit, globally distributed (if not with global coverage) compositional and thermophysical information has been gleaned from spectral images through the near-infrared to thermal wavelengths. However, these datasets are limited to resolutions much coarser than the scale of activity (many are 100+m/pixel, the best is CRISM with $18 \mathrm{~m} / \mathrm{pixel}$ ) and only capture surface exposures. In many areas, a thin layer of dust is enough to obscure the underlying layers. In such areas, geologic unit mapping can provide some constraints, based on extrapolation from visible outcrops, topography, and radar analysis. In situ compositional data, as collected by the Mars rovers and landers, allows for much more detailed measurement of surface and nearsubsurface properties, and coupling this with the global perspective provided through orbital observations has been key to constraining interpolative analyses.

Such analyses are of high importance for meteorological condition studies. Existing/continued orbital data provides a look at global circulation and atmospheric features, such as clouds. However, existing in situ aeolian and other meteorological data are insufficient to robustly answer surface-atmosphere interaction questions (as discussed in numerous community-generated reports $^{22,77}$ ). In situ monitoring of surface atmosphere interactions would provide key information for constraining volatile and sediment flux models under Mars' conditions [WP: Diniega et al.].

\section{Ties to technology, complementary research, and workforce investments}

To acquire needed new measurements, investment in landing technologies, especially for small spacecraft (landers or rovers) that could rideshare with Mars-focused and other launch opportunities in the next decade, would increase the opportunities to monitor and characterize a variety of environments. Advancements in small power systems (for generation and/or batteries) would enable monitoring through at least one full Mars year, and ideally also within higher latitudes. To increase the science value of new observations, a robust Mars Exploration Program could greatly enhance the ability to strategically link observations, especially between orbital and in situ assets [WP: Jakosky et al.]. A strong R\&A program is also needed to support data analysis and fundamental research studies that combine Mars' "natural laboratory" observations with laboratory/wind tunnel or field analog studies, for generation, testing, and calibration of the process models - and extension of these models to other planetary conditions. Finally, it is critical that the planetary science community and NASA foster an interdisciplinary, diverse, equitable, inclusive, and accessible environment so that cross-disciplinary and cross-target research is supported and a wide range of contributions can improve science advancement.

In Summary: To maximize the science return of Mars studies and advance our understanding of planetary surface processes and interpretation of geologic and climate histories, we need:

- Continued high-resolution, repeat imaging of the martian surface to identify and characterize surface changes and resultant landforms.

- In situ observation of active processes and the environmental drivers to develop detailed process models (that can be scaled for other planetary environments).

o Such new missions may be feasible with small spacecraft.

o Complementary wind-tunnel/laboratory and field analog studies are also needed.

- Coupled surface, subsurface, and atmospheric/meteorological measurements so as to enable "full system" studies (again, this aids scaling for other planetary environments).

o A robust Mars Program would improve coordination of observations of the Mars "system."

o A strong R\&A program and a diverse, equitable, inclusive, and accessible research environment would optimize analysis of such cross-disciplinary and cross-target data. 


\section{References (see the non-compressed list here):}

Color-coding highlights the range of planetary bodies with features and processes that could be/have been studied via comparative planetology with Mars as the basis: Inner planets/moons, Icy moons/small bodies, KBOs, comets, Mars, and extrasolar. References for fundamental physics or cross-planetary body studies are in black.

[1] Weitz et al. 1994 Icarus 112, 282-295. [2] Greeley et al. 1995 Icarus 115, 399-420. [3] Lorenz et al. 2006 Science 312, 724-727. [4] Barnes et al. 2015 Planet. Sci. 4, 1-19. [్] Jackson et al. 2020 JGR Planets 125, e2019JE006238. [6] Thomas et al. 2015 Science 347, aaa0440. [7] Telfer et al. 2018 Science 360 (6392), 992-997. [8] Claudin \& Andreotti 2006 Earth Planet. Sci. Lett. 252, 30-44. [9] Kok 2010 Phys. Rev. Lett. 104, 074502. [10] Kok et al. 2012 Rep. Prog. Phys. 75, 106901. [11] Duran Vinent 2019 Nat. Geosci. 12, 345-350. [12] Lapotre 2016 Science 353, 55-58. [13] Sullivan \& Kok 2017 JGR Planets 122, 2111-2143. [14] Vaz et al. 2017 Aeolian Res. 26, 101-116. [15] Monin \& Obukhov (1954 Contrib. Geophys. Inst. Acad. Sci. USSR, 151, e187. [16] Businger \& Yaglom 1971 Boundary-Layer Meteorology 2, 3-6. [17] Businger et al. 1967 J. Appl. Meteor. 6, 1025-1032. [18] Greeley et al. 1980 GRL 7, 121-124. [19] Greeley \& Iversen 1985 Cambridge Univ. Press: New York. [20] Cheng et al. 2013 Icarus 222, 808-817. [21] Iversen, \& White 1982 Sedimentology 29, 111-119. [22] MEPAG ICE-SAG Final Report 2019. [23] Diniega \& Smith 2020 Planet. Space Sci. 182, 104813. [24] Smith et al. 2018 Icarus 308, 2-14. [25] Boyce et al. 2012 Icarus, 221: 262-275. [26] Tornabene et al. 2012 Icarus 220, 348-368. [27] Blewett et al. 2011 Science 333, 1856-1859. [28] Thomas et al. 2014 Icarus 229, 221-235. [29] Denevi et al. 2012 Science 338 (6104), 246-249. [30] Sizemore et al. 2017. GRL 44: 6570-6578. [31] Moore et al. 2017 Icarus 287, 320-333. [32] Hofgartner et al. 2019 Icarus 334, 52-61. [33] Schenk et al. 2020 Icarus 113834. [34] Sunshine et al. 2016 JGR Planets 121, 2194-2210. [35] Vincent et al. 2015 Nature 523, 63-66. [36] Birch et al. 2017 Monthly Not. Royal Astro. Soc. 469, S50-S67. [37] Malliband et al. 2019 LPSC, 1804. [38] Krohn et al. 2014. Icarus 244, 120-132. [39] Scully et al. 2015. EPSL 411, 151-163. [40] Umurhan et al. 2016 AGU Fall Meeting, EP43D-08. [41] Piqueux et al. 2003 JGR 108, 5084. [42] Portyankina et al. 2017 Icarus 282, 93-103. [43] Mc Keown et al. 2017 Scientific Reports 7, 14181. [44] Thomas et al. 2010 Icarus 205, 296-310. [45] Kieffer 2007. JGR 112, E08005. [46] Soderblom et al. 1990 Science 250, 410-415. [47] Prockter et al. 1998 Icarus 135, 317-344. [48] Spencer \& Denk 2010 Science 327, 432-435. [49] Giese et al. 2008 Icarus 193, 359-371. [50] Cartwright et al. 2015 Icarus 257, 428-456. [51] Sori et al. 2017 Icarus 290, 1-13. [52] Howard \& Moore 2008 GRL 35 (3), L03203. [53] White et al. 2016 JGR Planets 121, 21-45. [54] Head \& Marchant 2003 Geology 31, 641-644. [55] Marchant et al. 1993 Geografiska Annaler 75A, 303-330. [56] Kreslavsky \& Head 2011 Icarus 216, 111-115. [57] Smith $20207^{\text {th }}$ Internat. Conf. Mars Polar Sci. Explor., 6040. [58] Turbet et al. 2018. Astron. Astrophy. 612, A86. [59] Malin et al. 2006 Science 314, 1573-1577. [60] Daubar et al. 2013 Icarus 225, 506-516. [61] Speyerer et al. 2016 Nature 538, 215-218. [62] Hartmann \& Daubar 2016 Meteoritics Planet. Sci. 52 (3), 493-510. [63] Suggs et al. 2014 Icarus 238, 23-36. [64] Spiga \& Smith 2018 Icarus 308, 197-208. [65] Hansen et al. 2015 Icarus 246, 183-191. [66] Zalucha \& Michaels 2013 Icarus 223 (2), 819-831. [67] Manning et al. 2019 Icarus 317, 509-517. [68] Buhler et al. 2019 Nature Astro., 1-8. [69] Trafton 1984 Icarus 58, 312-324. [70] Smith et al. 2020 Planet. Space Sci. 184, 104841. [71] Soto et al. 2015 Icarus 250, 553-569. [72] Bertrand et al. 2018 Icarus 309, 277-296. [73] Olkin et al. 2015 Icarus. 246, 220-225. [74] Hansen \& Paige (1994). [75] Lorenz et al. 1997 Science. 275, 642-644. [76] MEPAG NEX-SAG Report (2015). [77] MEPAG Goals Document (2020). 